\title{
The Aladin2 experiment: sensitivity study
}

\author{
G. Bimonte ${ }^{\mathrm{a}, 1}$ D. Born ${ }^{\mathrm{b}}$ E. Calloni ${ }^{\mathrm{a}}$ G. Esposito ${ }^{\mathrm{a}, 1}$ U. Hübner ${ }^{\mathrm{c}}$ \\ E. Il'ichev ${ }^{c}$ L. Rosa ${ }^{a}$ O. Scaldaferri ${ }^{a}$ F. Tafuri ${ }^{b}$ R. Vaglio ${ }^{a}$ \\ ${ }^{a}$ INFN, Sezione di Napoli, and Dipartimento di Scienze Fisiche, Complesso \\ Universitario di Monte S. Angelo, Via Cintia, Edificio N', 80126 Napoli, Italy \\ ${ }^{\mathrm{b}}$ Seconda Università di Napoli and INFN, Sezione di Napoli, Complesso \\ Universitario di Monte S. Angelo, Via Cintia, Edificio N', 80126 Napoli, Italy \\ ${ }^{\mathrm{c}}$ Institut Für Physikalische HochTechnologie e. V., Postfach 10 02 39 - 07r02 Jena, \\ Germany
}

\begin{abstract}
Aladin2 is an experiment devoted to the first measurement of variations of Casimir energy in a rigid body. The main short term scientific motivation relies on the possibility of the first demonstration of a phase transition influenced by vacuum fluctuations while, in the long-term and in the mainframe of the cosmological constant problem, it can be regarded as the first step towards a measurement of the weight of vacuum energy. In this paper, after a presentation of the guiding principle of the measurement, the experimental apparatus and sensitivity studies on final cavities will be presented.
\end{abstract}

Key words: Casimir energy, critical magnetic field

\section{Introduction}

Although phase transitions influenced by vacuum fluctuations are theoretically predicted to play a fundamental role in cosmology there is not, at present, an experimental demonstration of such a transition, not even at the microscopic scale. Furthermore, even if much progress has been made in the evaluation

${ }^{1}$ Partially supported by PRIN SINTESI

Preprint submitted to Elsevier Preprint and experimental verification of effects produced by vacuum energy in Minkowski space-time [1], it remains unclear why the observed universe exhibits an energy density much smaller than the one resulting from the application of quantum field theory and the equivalence principle [2]. Recently we pointed out that the sensitivity of present macroscopic small force detectors could allow, in a not too distant future, to experimentally verify the (passive) gravitational mass of vacuum energy, by detecting the extremely small forces 
exerted by the earth gravitational field on a multi-layer Casimir cavity whose vacuum energy were suitably modulated [3]. These considerations led our search for a method to modulate the Casimir energy in a rigid cavity without exchanging much more energy with the system (to avoid destroying the possibility of measuring and control): these conditions are satisfied if the cavity mirrors are composed by materials that can undergo a superconducting transition. In particular we showed that the use of phase transitions offers also the possibility to actually measure the energy change: for a given temperature, the external magnetic field needed to destroy superconductivity, i.e. the critical field, is in fact proportional to the total variation in free energy between the normal and superconducting state at zero field; if the condensation energy and Casimir variation are comparable, the total energy variation, and thus the critical field, of a film being part of a cavity can be sensibly different from that of a simple film [4]. The Aladin2 experiment has been conceived to verify this hypothesis, demonstrating the effect of vacuum fluctuations on a phase transition; the study of the possibility to modulate Casimir energy to verify its gravitational interaction, which was the original starting point, remains as a long-term motivation. The final measurement is foreseen for the end of 2007. Actually the cavity is placed at cryogenic temperature and an external magnetic field is applied, parallel to the plane of the films. The applied field $H^{C}(T)$ necessary to destroy superconductivity is mea- sured as a function of temperature. The expected signal is a different behavior of the function $H^{C}(T)$ with respect to the critical field $H^{F}(T)$ of a simple film. In this paper we report sensitivity studies on final film and cavities.

\section{Expected signal and experi- mental description}

The basic Casimir cavity is composed by a first thin layer $(10 \mathrm{~nm})$ of superconducting material $(\mathrm{Al})$ separated by an intermediate thin layer (10 nm of oxide) from a third metallic layer (100 nm), not superconducting. The areas used are $20 \times 20 \mu m^{2}$ and $100 \times 100 \mu m^{2}$ to verify that the effect does not depend on the area. The films and cavities are obtained by depositing the $\mathrm{Al}$ on the whole chip (some $\mathrm{cm}^{2}$ ) and growing films and cavities in the same way and in the same time (they both have a $10 \mathrm{~nm}$ oxide) while the only difference is on the final layer, deposited only for the cavities. Finally, the chip is divided into samples that contain both a film and a cavity that thus will experience the same field, reducing the alignment problem to a negligible contribution. The expected signal is shown in Fig. 1 where the difference in transition temperature $\delta(T)=T_{c}-T$ is reported as a function of the external magnetic field $H$. The upper curve refers to a simple film while the lower one to the same film being a mirror of the Casimir cavity. The theoretical predictions are valid from fields higher than a certain reduced 
critical field $H_{V} \approx 50$ Gauss, where the Casimir contribution to energy variation is simply a perturbation of condensation energy. Within these approximations the two curves are expected to show a constant difference $\Delta(T)=\delta(T)_{f}-\delta(T)_{c}$ (in our case $\Delta(T) \approx 0.2 m K)$, not dependent on the applied field.

In the region of field lower than $H_{V}$ the Casimir contribution to total energy variation cannot be considered a perturbation so that, strictly speaking, there is not a complete theoretical prediction and this is reported in Fig. 1 (see Proceedings in Nucl. Instr. Meth.) with the dotted part of the lower curve. The experimental apparatus is based on the commercial cryostat Oxford Instruments HELVLTD HelioxVL 3He, reaching the base temperature of $300 \mathrm{mK}$, inserted in a magnetically isolated dewar. The external field is generated by a 3.0 Gauss/mAmpere superconducting coil and the current is supplied and measured with a sensitivity better than $1 / 1000$ by a multimeter HP 34401A. The measurement method is a standard omodine four-wire resistance. The actual measurement is performed by fixing the external field and storing $R(T)$. A set of measurements is reported in Fig. 2 (see Proceedings in Nucl. Instr. Meth.): the transition width is approximately $50 \mathrm{mK}$.

The results of sensitivity study are displayed in Fig. 1 (see Proceedings in Nucl. Instr. Meth.) where $\delta(T)_{c}$ is reported as a function of the reduced field. It is seen that the sensitivity $\delta_{n}$ on the single measurement is approximatively $\delta_{n}=0.1 \mathrm{mK}$, which can be sufficient to perform the final measurement.

It is very important to stress that the actual measurement is not a measurement of the absolute behavior of the field of the cavity: such a measurement would require an accuracy (mostly on field-cavity alignment) which would impose extremely difficult experimental constraints. Instead, our measurement will be the difference of the behavior of two structures (film and cavity). In Fig. 1 (see Proceedings in Nucl. Instr. Meth.) the data are reported for the cavity case, while the final measurement simply would consist in repeating the measurement also for the film and to look for the eventual difference. Thus, the results reported if Fig. 1 (see Proceedings in Nucl. Instr. Meth.) must be regarded as sensitivity studies: if the film (upper curve) will show the expected $0.2 \mathrm{mK}$ shift in $\delta(T)$, our sensitivity should be sufficient to detect it.

\section{AC measurements}

As stated previously there is not a complete theory describing the behavior of $\delta(T)_{c}$ for applied field $\mathrm{H}$ near (or less than) $H_{V}$ : nonetheless this region corresponds to the physical region where the Casimir effect is predominant (i.e. it is not a perturbation of condensation energy), so that it is of particular interest from an experimental point of view. In this region, instead of studying the 
difference $\Delta(T)$ it is better to study the first derivative $\frac{d \delta(T)}{d H}$. In the case of a simple film the derivative is a straight line in the whole region of interest, while from general arguments [5] in the region of field $H<<H_{V}$, corresponding to transition temperatures very close to $T_{c}$, also for the cavity case the derivative must be linearly dependent on $H$.

Furthermore, as is already shown in Fig. 1 (see Proceedings in Nucl. Instr. Meth.), for $\mathrm{H}$ sufficiently high the difference of shift in temperature $\Delta(T)=\delta(T)_{f}-\delta(T)_{c}$ is expected to be constant (independent of $\mathrm{H}$ ), so that the two derivatives $\frac{d \delta(T)}{d H}{ }_{f, c}$ should converge to the same value. (We recall that the actual value of $\Delta(T)=0.2 m K$ in our case).

A possible situation is reported in Fig. 3 (see Proceedings in Nucl. Instr. Meth.). Whatever will be the actual curve, the relative difference of the two cases is expected to be quite high, of the order of some tens percent. Indeed, this corresponds to the fact that the difference $\Delta(T)=0.2 m K$ is expected to be reached already for $\frac{H}{H_{V}} \approx 3$ (where the shift in Casimir energy is a tenth of the condensation energy) where $\delta(T)_{f, c} \approx 0.6(0.4) m K$, respectively. From an experimental point of view this is very encouraging because, as shown in Fig. 3 (see Proceedings in Nucl. Instr. Meth.), the sensitivity is far better than few percent on each point, so that we expect to have the possibility to detect the vacuum fluctuation effect with a good signalto-noise ratio.

\section{References}

[1] M. Bordag, U. Mohideen and V.M. Mostepanenko, Phys. Rep. 353 (2001) 1.

[2] S. Weinberg, Rev. Mod. Phys. 61 (1989) 1.

[3] E. Calloni, L. Di Fiore, G. Esposito, L. Milano and L. Rosa, Phys. Lett. A 297 (2002) 328.

[4] G. Bimonte, E. Calloni, G. Esposito and L. Rosa Nucl. Phys. B 726 (2005) 441.

[5] M. Tinkham, Introduction to Superconductivity, Mc Graw-Hill, New York, 1996. 